\title{
STURM-LIOUVILLE PROBLEMS WITH EIGENPARAMETER DEPENDENT BOUNDARY CONDITIONS
}

\author{
by P. A BINDING*, P. J. BROWNE* and K. SEDDIGHI
}

(Received 26th June 1992)

Sturm theory is extended to the equation

$$
\left(-p y^{\prime}\right)^{\prime}+q y=\lambda r y
$$

for $1 / p, q, r \in L_{1}[0,1]$ with $p, r>0$, subject to boundary conditions

and

$$
\left(a_{j} \lambda+b_{j}\right) y(j)=\left(c_{j} \lambda+d_{j}\right)\left(p y^{\prime}\right)(j),
$$

$$
(-1)^{j} \delta_{j} \leqq 0 \text { where } \delta_{j}=a_{j} d_{j}-b_{j} c_{j}, \quad j=0,1 \text {. }
$$

Oscillation and comparison results are given, and asymptotic estimates are developed. Interlacing of eigenvalues with those of a standard Sturm-Liouville problem where the boundary conditions are $a_{j}, y(j)=$ $c_{j}\left(p y^{\prime}\right)(j), j=0,1$, forms a key tool.

1991 Mathematics subject classification: 34B24.

\section{Introduction}

A large body of literature has built up, over the years, on problems of SturmLiouville type but where the boundary conditions depend affinely on $\lambda$. Both Walter [12] and Fulton [4] have extensive bibliographies and we also refer to Fulton for various physical applications. Most of the work these authors cite deals with the case of one fixed and one variable end condition, say $a_{0}=c_{0}=0$ and $\delta_{1}>0$ in the notation of the abstract. Such work also deals almost entirely with completeness and expansion theory in $L_{2}[0,1] \oplus \mathbb{C}^{k}$, where $k$ is the number of $\lambda$-dependent boundary conditions, although Fulton also treats asymptotics, to which we return below. Completeness and expansion theory is also the focus of more recent works, e.g. [6], [8] and their references, some of which cover general settings and, in particular, dispense with the 'right definiteness' conditions $\delta_{1}>0, r>0$.

Problems with $\lambda$-dependent end conditions can also be found in the literature on the calculus of variations: see Reid [10, p, 399] for references treating various topics (but not Sturm theory). Nevertheless Reid $[10, \S \mathrm{V} .7]$ shows how focal point analysis can be

\footnotetext{
* Research support in part by grants from the NSERC of Canada.
} 
used to give an oscillation theorem in the case when $(-1)^{j}\left(p y^{\prime} / y\right)(j)$ is non-increasing in $\lambda$. This corresponds to the case $c_{j}=0$ which we call "exceptional" and which we treat in Section 5. We remark that Reid's methods and ours both permit nonafine boundary conditions, but for simplicity we adhere to the well studied formulation above. As Reid notes in [11, p. 48], the oscillation theorem for the case $c_{j}=0$ also follows from classical arguments involving Picone's identity: see also Ince $[7, \S 10.6]$. It is worth pointing out that the "left definite" fixed end condition problem can be transformed to this case [10, p. 251]. The corresponding variable end condition problem requires further analysis, however, and we intend to pursue this subsequently.

We have two basic aims here. One is to show how known properties of the Prüfer transformation, together with simple geometrical arguments, yield a comprehensive Sturm theory for variable end condition problems with $(-1)^{j} \delta_{j} \leqq 0$. The other is to establish a framework for subsequent work on the more difficult cases where such definiteness conditions fail. We operate for the most part under the minimal coefficient conditions, $1 / p, q, r \in L_{1}[0,1]$. Thus even in the exceptional case $c_{j}=0$, our work generalizes that of, say, Reid $[10, \S \mathrm{V} .7]$ where $p, q, r$ are assumed to be continuous.

In Section 2 we set up our framework and we apply it to the usual fixed end condition problem. Section 3 contains the basic results for one variable end condition with $c_{1} \neq 0$. The eigenvalues $\lambda_{n}$ interlace those of the "asymptotic" fixed end condition problem obtained by formally setting $\lambda=\infty$ in the $\lambda$-dependent boundary condition. This leads to an asymptotic estimate of the form

$$
\lambda_{n}=\left(n \pi / \int_{0}^{1} \sqrt{r / p}\right)^{2}+o\left(n^{2}\right)
$$

A modified Prüfer transformation gives a better estimate if $p r$ is absolutely continuous, and this includes Fulton's results for $p=r \equiv 1$. Comparison principles parallel those for the usual (fixed end condition) Sturm-Liouville problem, but the oscillation theorem needs modifying: one oscillation count (which we identify) corresponds to two distinct eigenvalues. In Section 4 these results are generalized to two variable end conditions: this time either two oscillation counts have two eigenvalues each, or else one count has three.

Section 5 contains the corresponding results for the exceptional cases $c_{j}=0$ (noted above) and $\delta_{j}=0$. The latter have been dismissed by previous authors as equivalent to fixed end condition problems, but we shall show that the results are, in fact, parallel to those for $(-1)^{v} \delta_{j}<0$. We conclude with an Appendix on asymptotics for the fixed end condition (weighted Sturm-Liouville) problem with $p r \in A C[0,1]$ and $q \in L_{1}[0,1]$. The literature abounds with asymptotic developments for the eigenvalues of the unweighted problem under various conditions on $q$, cf. $[1, \S 12.8],[5, \S 8.4],[7, \S 11.4],[9$, p. 35] . We were unable to locate such results in the generality we needed and hope, therefore, that the Appendix may be of interest in its own right.

\section{Preliminaries}

We study the differential equation 


$$
-\left(p y^{\prime}\right)^{\prime}+q y=\lambda r y, \quad '=\frac{d}{d x}
$$

on the interval $[0,1]$, assuming $1 / p, q, r \in L_{1}[0,1]$ with $p, r>0$. Until Section 4 , we impose an initial condition of the form

$$
b_{0} y(0)=d_{0}\left(p y^{\prime}\right)(0),\left(b_{0}, d_{0}\right) \neq(0,0) .
$$

This enables us to define a Prüfer angle $\theta(\lambda, x)$ by means of the differential equation

$$
\theta^{\prime}=(1 / p) \cos ^{2} \theta+(\lambda r-q) \sin ^{2} \theta
$$

with initial condition

$$
\theta(\lambda, 0)=\cot ^{-1}\left(b_{0} / d_{0}\right)
$$

which we assume to be in the interval $[0, \pi[$. Standard results on differential inequalities then show that $\theta(\lambda, 1)$ is a continuous strictly increasing function of $\lambda$ and Atkinson [1, §8.4] establishes the limits

$$
\theta(\lambda, 1) \rightarrow 0 \text { as } \lambda \rightarrow-\infty, \theta(\lambda, 1) \rightarrow \infty \text { as } \lambda \rightarrow \infty .
$$

We define the right-hand Dirichlet problem (RDP) associated with $(2.1,2.2)$ by appending the boundary condition $y(1)=0$. The corresponding eigenvalues are denoted by $\lambda_{n}^{D}, n=0,1,2, \ldots$. The above results can be translated as follows into facts about the graph of $f(\lambda):=\cot \theta(\lambda, 1)$.

Theorem 2.1. The graph $\mu=f(\lambda)$ consists of countably many continuous branches $B_{n}$, $n=0,1,2, \ldots$. Interpreting $\lambda_{-1}^{D}$ as $-\infty$, we have for $n \geqq 0$ :

(1) $\theta(\lambda, 1) \in] n \pi,(n+1) \pi[$ for $\lambda \in] \lambda_{n-1}^{D}, \lambda_{n}^{D}\left[\right.$ and $\theta\left(\lambda_{n}^{D}, 1\right)=(n+1) \pi$

(2) $B_{n}$ is defined for $\left.\lambda \in\right] \lambda_{n-1}^{D}, \lambda_{n}^{D}[$ and $f$ decreases over this interval with $\lim _{\lambda \nmid \lambda_{n-1}^{D}} f(\lambda)=+\infty, \lim _{\lambda \uparrow \lambda_{n}^{D}} f(\lambda)=-\infty$.

(3) $\lambda=\lambda_{n}^{D}$ are the vertical asymptotes of the graph of $f$.

The following result, which states (roughly) that abscissae $\lambda$ from $B_{n}$ correspond to eigenfunctions with $n$ internal zeros, illustrates the appropriateness of using $\cot \theta(\lambda, 1)$ rather than the customary $\tan \theta(\lambda, 1)$.

Lemma 2.2. If $n \geqq 0$ and $\left.\lambda \in] \lambda_{n-1}^{D}, \lambda_{n}^{D}\right]$ then all solutions of (2.1), (2.2) possess exactly $n$ zeros in $] 0,1[$.

Proof. The solutions of (2.1), (2.2) form a vector space of dimension 1. The arguments of Atkinson [1, p. 209] show that $\theta(\lambda, \cdot)$ increases through any value $x_{0}$ at which $\theta\left(\lambda, x_{0}\right)$ is an integer multiple of $\pi$. Since $\left.\left.\theta(\lambda, 1) \in\right] n \pi,(n+1) \pi\right]$, there is no 
$x \in] 0,1[$ such that $\theta(\lambda, x)=(n+1) \pi$. Since $\theta(\lambda, 0) \in[0, \pi[$, the result follows directly for $n=0$, while for $n>0$ we have $\theta(\lambda, x)=n \pi$ for some $x \in] 0,1[$.

We are now ready to discuss general right hand boundary conditions. The simplest case is the fixed end condition (Sturm-Liouville) problem.

Corollary 2.3. If the solutions of (2.1), (2.2) are also required to satisfy $\theta(\lambda, 1) \equiv$ $\beta(\bmod \pi), \beta \in] 0, \pi]$, then for each $n \geqq 0$ there is a unique eigenvalue $\lambda=\lambda_{n}$ whose corresponding eigenfunction $y$ has $n$ zeros in $] 0,1\left[\right.$. The $\lambda_{n}$ interlace the $R D P$ eigenvalues $\lambda_{n}^{D}$, in the sense that

$$
\lambda_{n} \leqq \lambda_{n}^{D}<\lambda_{n+1}, \quad n=0,1, \ldots
$$

Proof. If $\beta \neq \pi$ then the horizontal line $\mu=\cot \beta$ cuts $B_{n}$ at $\left(\lambda_{n}, \beta\right)$ say, and if $\beta=\pi$ we set $\lambda_{n}=\lambda_{n}^{\mathrm{D}}$. In both cases Lemma 2.2 applies.

This includes the usual Sturm oscillation theorem, and we turn now to the analogous procedure for the variable end condition

$$
\left(a_{1} \lambda+b_{1}\right) y(1)=\left(c_{1} \lambda+d_{1}\right)\left(p y^{\prime}\right)(1)
$$

Until Section 5 we assume $c_{1} \neq 0, \delta_{1}:=a_{1} d_{1}-b_{1} c_{1}>0$. Evidently (2.6) may be written as

where

$$
\cot \theta(\lambda, 1)=g(\lambda)
$$

$$
g(\lambda):=\left(a_{1} \lambda+b_{1}\right) /\left(c_{1} \lambda+d_{1}\right) \text {. }
$$

Lemma 2.4. The graph $\mu=g(\lambda)$ is a hyperbola with horizontal asymptote $\mu=a_{1} / c_{1}$ and vertical asymptote $\lambda=-d_{1} / c_{1}$, and $g$ increases along both branches.

Indeed we have the expression

$$
g(\lambda)=\left(a_{1} / c_{1}\right)-\delta_{1} c_{1}^{-2}\left(\lambda+d_{1} / c_{1}\right)^{-1} .
$$

We now define $\left.\left.\alpha_{1} \in\right] 0, \pi\right]$ by

$$
\cot \alpha_{1}=a_{1} / c_{1}
$$

Definition 2.5. The right hand asymptotic problem (RAP) associated with (2.1), (2.6) is defined by the equation (2.1), the condition (2.2) and the condition

$$
a_{1} y(1)=c_{1}\left(p y^{\prime}\right)(1)
$$

The corresponding eigenvalues are denoted by $\lambda_{n}^{A}$. 
This standard Sturm-Liouville problem is one of our main tools in the next section. We set

$$
\begin{gathered}
\theta^{-}=\theta-\alpha_{1}, \\
g^{-}(\lambda)=\cot \theta^{-}(\lambda, 1)
\end{gathered}
$$

using (2.7) and $\cot \theta(\lambda, 1)=\left(p y^{\prime}\right)(1) / y(1)$. Then a simple calculation yields

$$
g^{-}(\lambda)=\delta_{1}^{-1}\left[\left(a_{1}^{2}+c_{1}^{2}\right) \lambda+a_{1} b_{1}+c_{1} d_{1}\right] .
$$

This translation of $\theta$ to $\theta^{-}$converts the graph of $g$ to a straight line (the graph of $g^{-}$), which is what we would have obtained from (2.7) were $c_{1}=0$. The graph of $f$ is also changed by this translation and the following is the analogue of Theorem 2.1 for $f^{-}(\lambda)=\cot \theta^{-}(\lambda, 1)$ defined this time by means of (2.3), (2.4).

Corollary 2.6. The graph of $\mu=f^{-}(\lambda)$ is qualitatively similar to that of $f$ except that $\lim _{\lambda \rightarrow-\infty} f^{-}(\lambda)=-a_{1} / c_{1}$ and the vertical asymptotes are $\lambda=\lambda_{n}^{A}, n=0,1, \ldots$.

\section{One variable end condition}

In this section we study the problem (2.1), (2.2), (2.6) assuming $c_{1} \neq 0, \delta_{1}>0$. For the oscillation theorem, we suppose that the vertical asymptote for $g$, (Lemma 2.4), intersects the $N$ th branch $B_{N}$ of $f$ or is its right hand asymptote, (Theorem 2.1); i.e. we select $N$ so that

$$
\lambda_{N-1}^{D}<-d_{1} / c_{1} \leqq \lambda_{N}^{D}
$$

Theorem 3.1. The eigenvalues $\lambda=\lambda_{n}$ can be ordered $\lambda_{0}<\lambda_{1}<\ldots$ where for $n \leqq N$ the corresponding eigenfunction $y$ has $n$ zeros in $] 0,1[$, and for $n>N, y$ has $n-1$ such zeros.

Proof. The $\lambda_{n}$ correspond to the intersection points of the graphs of $f$ and $g$. Evidently, the left branch of the graph of $g$ intersects each $B_{n}$ precisely once for $0 \leqq n \leqq N$, and Lemma 2.2 gives the corresponding oscillation counts. A similar argument holds for $n>N$ using the right branch for $g$, unless $-d_{1} / c_{1}=\lambda_{N}^{D}$. In this latter case, $\lambda_{N+1}=\lambda_{N}^{D}$ and again the eigenfunction has $N$ zeros by Lemma 2.2 .

We turn next to the comparison theorem and suppose that the coefficients in (2.1), (2.6) depend on a parameter $t$.

Theorem 3.2. (i) If $a_{1} / c_{1}$ and $1 / p$ are non increasing, $b_{0} / d_{0}$ and $q$ are non decreasing and $r, \delta_{1}, c_{1}$ and $d_{1}$ are constant in $t$, then each $\lambda_{n}$ is nondecreasing in $t$. 
(ii) If the assumptions of (i) hold except that $r$ and $\delta_{1}$ are nondecreasing in $t$ then so is $\lambda_{n}$ for all $n$ such that $\lambda_{n}>\max \left\{0,-d_{1} / c_{1}\right\}$.

Proof. (i) By differential inequality theory, $f(\lambda)$ is nondecreasing in $t$ for fixed $\lambda$. By (2.8), $g(\lambda)$ is nonincreasing, so the conclusion follows from the piecewise monotonicity of $f(\lambda)$ and $g(\lambda)$ in $\lambda$ given in Theorem 2.1 and Lemma 2.4.

(ii) The same argument holds for $\lambda>\max \left\{0,-d_{1} / c_{1}\right\}$.

Remark. In a similar way we see that if the coefficients are continuous in $t$, then so are the $\lambda_{n}$.

Theorem 3.1 leads to a partial interlacing of $\lambda_{n}$ and $\lambda_{n}^{D}$, but complete interlacing is obtained if we use the $\lambda_{n}^{A}$ of Definition 2.5 instead, or if we consider only $n>N$.

Theorem 3.3. (i) $\lambda_{n-1}^{A}<\lambda_{n}<\lambda_{n}^{A}$ for all $n$.

(ii) $\lambda_{n-1}^{D}<\lambda_{n}^{A}<\lambda_{n}<\lambda_{n}^{D}$ for all $n>N$.

Proof. (i) The $\lambda_{n}$ also correspond to the intersection points of the graphs of $f^{-}$and $g^{-}$, and it is evident that the latter, being a straight line of positive slope by (2.11), intersects each branch of the former precisely once. The result thus follows from Corollary 2.6.

(ii) This follows by considering the graphs of $f$ (Theorem 2.1) and $g$ (Lemma 2.4) for $\lambda>-d_{1} / c_{1}$.

Corollary 3.4. $\lambda_{n}=(n \pi / \sigma)^{2}+o\left(n^{2}\right)$ as $n \rightarrow \infty$, where $\sigma=\int_{0}^{1} s$ and $s=(r / p)^{1 / 2}$.

Proof. This follows from Theorem 3.3 and the corresponding estimate for $\lambda_{n}^{A}$, [2]. Note that $p^{-1 / 2}, r^{1 / 2} \in L_{2}[0,1]$ guarantee $s \in L_{1}[0,1]$.

The graphs of $f^{-}$and $g^{-}$suggest that $\lambda_{n}-\lambda_{n-1}^{\lambda} \downarrow 0$ as $n \rightarrow \infty$, which under appropriate conditions will improve the asymptotic estimates. We shall now examine the relation between $\lambda_{n}$ and $\lambda_{n-1}^{A}$ more carefully. We introduce a modified Prüfer angle by

$$
\cot \phi=(\lambda s)^{-1 / 2} y^{\prime} / y=(\operatorname{pr} \lambda)^{-1 / 2} \cot \theta .
$$

For related, but slightly different, transformations see $[2$, p. 385$],[3, \S 4.1],[5, \S 8.4]$.

Theorem 3.5. If $p r \in A C[0,1]$ then $\lambda_{n}-\lambda_{n-1}^{A}=0\left(n^{-2}\right)$, as $n \rightarrow \infty$.

Proof. From (3.2), a simple calculation gives

$$
\phi^{\prime}=(\lambda r / p)^{1 / 2}-(\lambda p r)^{-1 / 2} q \sin ^{2} \phi+\left(p r^{\prime}\right)(4 p r)^{-1} \sin 2 \phi,
$$


where the right side obeys a Carathéodory condition in $(\phi, x)$ and is smooth in $\phi$ and $\lambda(>0)$. The initial condition may be taken from (2.4) and (3.2) in the form

$$
\cot \phi(\lambda, 0)=((p r)(0) \lambda)^{-1 / 2} b_{0} / d_{0} .
$$

Denoting $\partial \cdot / \partial \lambda$ by $(\cdot)_{\lambda}$ we may therefore apply $[10, \S$ II.4] to yield

$$
2 \phi_{\lambda}^{\prime}=\lambda^{-1 / 2}\left[s+o_{1}(1)\right]+\phi_{\lambda}\left[(p r)^{\prime}(p r)^{-1} \cos 2 \phi+o_{1}(1)\right]
$$

where $o_{1}(1) \rightarrow 0$ in $L_{1}[0,1]$ as $\lambda \rightarrow \infty$. The integrating factor

$$
h(x)=\exp \left\{\frac{1}{2} \int_{x}^{1}\left((p r)^{\prime}(p r)^{-1} \cos 2 \phi+o_{1}(1)\right)\right\}
$$

for (3.5) thus satisfies $\gamma \leqq h(x) \leqq \gamma^{-1}$ for some positive constant $\gamma$, so

$$
\begin{aligned}
\phi_{\lambda}(1)-h(0) \phi_{\lambda}(0) & =\frac{1}{2} \lambda^{-1 / 2} \int_{0}^{1}\left[s+o_{1}(1)\right] h \\
& \geqq \frac{1}{2} \gamma \lambda^{-1 / 2} \sigma+o\left(\lambda^{-1 / 2}\right) \\
& \geqq \frac{1}{4} \gamma \lambda^{-1 / 2} \sigma \text { for } \lambda \text { large enough. }
\end{aligned}
$$

Now

$$
\begin{aligned}
\phi_{\lambda}(0) & =-\left(\operatorname{cosec}^{2} \phi(\lambda, 0)\right)^{-1}(\cot \phi(\lambda, 0))_{\lambda} \\
& =-\left(1+0\left(\lambda^{-1}\right)\right)^{-1} 0\left(\lambda^{-3 / 2}\right)=0\left(\lambda^{-3 / 2}\right)
\end{aligned}
$$

by (3.4), so (3.7) gives

$$
\phi_{\lambda}(1) \geqq \delta \lambda^{-1 / 2} \text { for some constant } \delta>0 .
$$

The variational equation for (2.3) shows that $\theta(\lambda, 1)$ is $C^{1}$ in $\lambda(>0)$, so by Theorem 3.3(ii), $f$ is $C^{1}$ on $I_{n}:=\left[\lambda_{n-1}^{A}, \lambda_{n}\right]$. Thus by the mean value theorem

$$
f\left(\lambda_{n}\right)-f\left(\lambda_{n-1}^{A}\right)=\left(\lambda_{n}-\lambda_{n-1}^{A}\right) f_{\lambda}(\lambda)
$$

for some $\lambda \in I_{n}$. From (2.8) the left side is $0\left(\lambda_{n}^{-1}\right)=0\left(n^{-2}\right)$ by Corollary 3.4, so it is enough to show that, for some $\varepsilon>0$,

$$
f_{\lambda}(\lambda) \leqq-\varepsilon \text { for all } \lambda \in I_{n} \text { and } n \text { large. }
$$

We claim

$$
f(\lambda)=0(1)
$$


for such $\lambda$. Indeed, (2.8) gives $f\left(\lambda_{n}\right)=0(1),(2.10)$ gives $f\left(\lambda_{n-1}^{A}\right)=0(1)$ and so Theorems 2.1 and 3.3(ii) show that $f$ decreases continuously over $I_{n}$. Now with $\rho^{2}=(p r)(1)$ we obtain from (3.2) the required estimate

$$
\begin{aligned}
f_{\lambda}(\lambda) & =\left(\rho \lambda^{1 / 2} \cot \phi(\lambda, 1)\right)_{\lambda} \\
& =\frac{1}{2} \rho \lambda^{-1 / 2} f(\lambda)-\rho \lambda^{1 / 2}\left(\operatorname{cosec}^{2} \phi(\lambda, 1)\right) \phi_{\lambda}(1) \\
& \leqq-\delta \rho+0\left(\lambda^{-1 / 2}\right)
\end{aligned}
$$

by (3.8) and (3.9), since $\operatorname{cosec}^{2} \phi(\lambda, 1)=1+\cot ^{2} \phi(\lambda, 1)=1+\rho^{-2} \lambda^{-1} f(\lambda)^{2}=0(1)$, again by (3.9).

Corollary 3.6. (i) If $p r \in A C[0,1]$, then $\lambda_{n}=((n+v) \pi / \sigma)^{2}+o(n)$ where $v=-\frac{1}{2}$ if $d_{0}=0$, $v=-1$ if $d_{0} \neq 0$.

(ii) If in addition (pr)' $\in A C[0,1]$ then $o(n)$ may be replaced by $0(1)$.

(iii) If pr is a constant $\left(\rho^{2}\right)$ then $O(1)$ may be estimated by

$$
R=(\rho \sigma)^{-1}\left(-2\left[\cot ^{*} \theta(\lambda, j)\right]_{j=0}^{j=1}+\int_{0}^{1} q\right)+o(1)
$$

where $\cot ^{*} \theta=\cot \theta$ if $\theta \neq 0, \cot ^{*} 0=0$, and $\theta(\lambda, j)$ are determined by (2.4), (2.10).

This follows immediately from Theorem A3 in the Appendix. We remark that in the case $p=r \equiv 1$ (so $\rho=\sigma=1$ ), we recover Fulton's formulae [4, $\S 4$, Cases 1 and 2].

\section{Two variable end conditions}

In this section we consider the variable end condition problem (VEP) defined by the equation (2.1) subject to the boundary conditions

$$
\left(a_{j} \lambda+b_{j}\right) y(j)=\left(c_{j} \lambda+d_{j}\right)\left(p y^{\prime}\right)(j), \quad j=0,1,
$$

with $c_{j} \neq 0$ and $(-1)^{j} \delta_{j}<0$. We define $\alpha_{0} \in[0, \pi[$ by

$$
\cot \alpha_{0}=a_{0} / c_{0}
$$

and $\theta(\lambda, 0) \in] \alpha_{0}, \alpha_{0}+\pi[$ by

$$
\cot \theta(\lambda, 0)=\left(a_{0} \lambda+b_{0}\right)\left(c_{0} \lambda+d_{0}\right)^{-1} .
$$

We now define $\lambda_{n}^{D}, \lambda_{n}^{A}, f(\lambda)$ and $f^{-}(\lambda)$ as in Section 2 but using (4.3) in place of (2.4). In particular, $\lambda_{n}^{D}$ and $\lambda_{n}^{A}$ are eigenvalues for problems with variable left end conditions, and Section 3 applies to these problems provided we replace $x$ by $1-x$. 
Theorem 4.1. Theorem 2.1 carries over the present case, where the initial conditions are given by (4.3).

Proof. On each of its branches, $\cot ^{-1}$ is decreasing as is the right side of (4.3) on both of its branches. Further

$$
\lim _{\lambda \uparrow-d_{0} / c_{0}} \cot \theta(\lambda, 0)=-\infty, \lim _{\lambda \downarrow-d_{0} / c_{0}} \cot \theta(\lambda, 0)=+\infty
$$

so recalling that $\theta(\lambda, 0) \in] \alpha_{0}, \alpha_{0}+\pi[$, we see that $\theta(\lambda, 0)$ increases through $\pi$ as $\lambda$ increases through $-d_{0} / c_{0}$. It follows that $\theta(\lambda, 0)$ increases for all real $\lambda$.

Differential inequality theory applied to (2.3) now shows that $\theta(\lambda, 1)$ increases for all real $\lambda$. Similarly, $\alpha_{0}<\theta(\lambda, 0)$ and (2.5) yield $\theta(\lambda, 1) \rightarrow \infty$ as $\lambda \rightarrow \infty$. Finally, $\theta(\lambda, 0) \rightarrow \alpha_{0}$ as $\lambda \rightarrow-\infty$, so $\theta(\lambda, 1) \rightarrow 0$ by (2.5) and continuous dependence theory for (2.3). Thus $\theta$ has all the properties needed for the proof of Theorem 2.1.

In order to state the oscillation theorem, we define the double Dirichlet problem to be the equation (2.1) subject to Dirichlet conditions $y(j)=0, j=0,1$. The eigenvalues of this problem are denoted by ${ }^{D} \lambda_{n}^{D}$. We now define indices $N(j)$ by

$$
{ }^{D} \lambda_{N(j)-1}^{D}<-d_{j} / c_{j} \leqq{ }^{D} \lambda_{N(j)}^{D}, \quad j=0,1,
$$

cf. (3.1): as before we take ${ }^{D} \lambda_{-1}^{D}=-\infty$. Finally we write

$$
m=\min _{j=0,1} N(j), M=\max _{j=0,1} N(j)
$$

Theorem 4.2. The eigenvalues of the VEP may be ordered $\lambda_{0}<\lambda_{1}<\ldots$ where the corresponding eigenfunction has $n$ zeroes in $] 0,1[$ for $n \leqq m, n-1$ zeros for $m<n \leqq M+1$ and $n-2$ for $n>M+1$.

Proof. Suppose $m=N(1)$. As in Theorem 3.1, the $\lambda_{n}$ correspond to the intersection points of the graphs of $f$ and $g(2.8)$, and each branch $B_{n}$ has exactly one such point except for $B_{N}$, say, which has two. Here $N$ is specified by

$$
\lambda_{N-1}^{D}<-d_{1} / c_{1} \leqq \lambda_{N}^{D}
$$

cf. (3.1).

Replacing $x$ by $1-x$ we now view the RDP (with eigenvalues $\lambda_{n}^{D}$ ) as one of the type considered in Section 3. As in Theorem 3.1 the $\lambda_{n}^{D}$ are the intersections of the graph of an $f$ generated by a problem with an initial Dirichlet condition and a $g$ generated by the condition

$$
\left(a_{0} \lambda+b_{0}\right) y(1)=-p(0) y^{\prime}(1)\left(c_{0} \lambda+d_{0}\right)
$$


Thus for $n \leqq N(0)$, (respectively, $n>N(0)$ ), the eigenfunction corresponding to $\lambda_{n}^{D}$ has $n$, (respectively, $n-1)$ zeroes in ]0, 1[: see (4.4). Moreover $N=N(1)$ by (4.5) so for $n \leqq N(1)$, the eigenfunctions of the VEP and RDP have the same number of internal zeros, and for $n>N(1)$, the RDP eigenfunction has one more zero than that of the VEP. The argument for the case $m=N(0)$ is analogous.

The comparison theorem is similar to Theorem 3.2 except that variations in $a_{0}$ and $c_{0}$ are now possible. Again we suppose that our coefficient functions depend on a parameter $t$.

Theorem 4.3. (i) If $a_{1} / c_{1}$ and $1 / p$ are nonincreasing, $a_{0} / c_{0}$ and $q$ are nondecreasing and $r, \delta_{j}, c_{j}$ and $d_{j}(j=0,1)$ are constant in $t$, then each $\lambda_{n}$ is nondecreasing in $t$.

(ii) If the assumptions of (i) hold except that $r$ and $(-1)^{j} \delta_{j}$ are nonincreasing in $t$, then so are all positive $\lambda_{n}$ for $n>M$.

For the interlacing theorem we introduce one more related problem. The SturmLiouville double asymptotic problem, with eigenvalues ${ }^{A} \lambda_{n}^{A}$, is defined by the equation (2.1) and the end conditions

$$
a_{j} y(j)=c_{j}\left(p y^{\prime}\right)(j), \quad j=0,1 .
$$

Theorem 4.4. $\quad A \lambda_{n-2}^{A}<\lambda_{n}<\lambda_{n}^{A}, \quad n=0,1, \ldots$

Proof. From the proof of Theorem 4.1, we claim Corollary 2.6 and hence Theorem 3.3(i) as stated (but now for variable initial condition). Replacing $x$ by $1-x$ we have ${ }^{A} \lambda_{n-1}^{A}<\lambda_{n}^{A}<{ }^{A} \lambda_{n}^{A}$ from Theorem 3.3(i) and the conclusion is now immediate.

For large, $n$, Theorem 4.4 can be improved: in fact we have the following immediate consequence of Theorem 3.5 and the reasoning of Theorem 4.2.

Corollary 4.5. If $p r \in A C[0,1]$ then

$$
\lambda_{n}={ }^{A} \lambda_{n-2}^{A}+0\left(n^{-2}\right) \text { as } n \rightarrow \infty .
$$

Thus the asymptotic estimates of Corollary 3.6 carry over to the present situation, except that $v=-2$ in all cases (since $c_{j} \neq 0$ ensures that (4.6) are not of Dirichlet type) and $\theta(\lambda, j)$ are now determined by (4.6).

\section{Exceptional cases}

We now consider the possibility $c_{j} \delta_{j}=0$ starting with one variable end condition. We note that Corollary 2.3 shows that the usual (Sturm) oscillation theorem is valid in the case $a_{1}=c_{1}=0$. The following shows that in other cases where $\delta_{1}=0$ and (2.6) is a 
genuine boundary condition, the oscillation theorem generally takes the form of Theorem 3.1 instead. Recall the definition of the RAP and RDP from Section 2.

Theorem 5.1. For the Problem (2.1), (2.2), (2.6) suppose $\delta_{1}=0$, but that $\left(a_{1}, c_{1}\right) \neq(0,0)$. Then (i) Theorem 3.1 holds for an appropriate index $N$, unless there is a repeated eigenvalue in the sense that $-d_{1} / c_{1}$ is a RAP eigenvalue if $c_{1} \neq 0$ or $-b_{1} / a_{1}$ is a RDP eigenvalue if $c_{1}=0$. (ii) If there is a repeated eigenvalue then Sturm's oscillation theorem holds.

Proof. (i) If $c_{1} \neq 0$, then $b_{1}=a_{1} d_{1} / c_{1}$ and (2.6) reads

$$
c_{1}^{-1} a_{1}\left(c_{1} \lambda+d_{1}\right) y(1)=\left(c_{1} \lambda+d_{1}\right)\left(p y^{\prime}\right)(1),
$$

from which either $a_{1} y(1)=c_{1}\left(p y^{\prime}\right)(1)$ or $c_{1} \lambda+d_{1}=0$. An eigenvalue $\lambda_{n}$ thus either comes from the (Sturm-Liouville) RAP, or else equals $-d_{1} / c_{1}$. Hence $N$ is defined by $\lambda_{N-1}^{A}<-d_{1} / c_{1}<\lambda_{N}^{A}$.

If $c_{1}=d_{1}=0 \neq a_{1}$, then

$$
\left(a_{1} \lambda+b_{1}\right) y(1)=0
$$

from which an eigenvalue $\lambda_{n}$ either comes from the (Sturm-Liouville) RDP or else equals $-b_{1} / a_{1}$. Hence $N$ is specified by $\lambda_{N-1}^{D}<-b_{1} / a_{1}<\lambda_{N}^{D}$.

(ii) In this case we have coalescence of either $-d_{1} / c_{1}$ with $\lambda_{N}^{A}$ or $-b_{1} / a_{1}$ with $\lambda_{N}^{D}$. The correspopnding eigenfunctions satisfy (2.1) and (2.2) and hence are unique up to scalar multiplication, so there is no "extra" eigenfunction in this case.

Remark. The discrepancy between (i) and (ii) appears because Sturm's theorem includes only eigenfunctions. A result like (i) is always available of one includes a "generalized" eigenfunction $z$ satisfying $-\left(p z^{\prime}\right)^{\prime}+(q-\lambda r) z=r y_{N}$ with $z(0)=\left(p z^{\prime}\right)(0)=0$, where span $\left(y_{N}, z\right)$ plays the role of the algebraic eigenspace at $\lambda_{N}$ in case (ii).

In the situation of Theorem 5.1, the remaining theorems of Section 3 require simple modifications which will be left to the reader. We turn now to the case $\delta_{1} \neq 0, c_{1}=0$.

Corollary 5.2. If $c_{1}=0<\delta_{1}$ then Sturm's oscillation theorem holds.

Proof. In this case, the graph of $g(2.7)$ is a straight line of positive slope, so the proof of Corollary 2.3 remains valid.

As noted in the Introduction, this result can be found for continuous coefficients in various sources; cf[11, p. 48]. Indeed it suffices for $g$ to be a nondecreasing function. The comparison Theorem 3.2 requires obvious modification, the interlacing Theorem 3.3 remains valid since the RAP and the RDP coincide, and hence Corollary 3.4 is also unchanged. The asymptotic Theorem 3.5 now needs modification, however, since it is no longer true in general that $\lambda_{n}-\lambda_{n-1}^{A} \rightarrow 0$ as $n \rightarrow \infty$. 
Theorem 5.3. If $c_{1}=0<\delta_{1}$ and $p r \in A C[0,1]$, then $\lambda_{n}-\lambda_{n-1}^{A}=0(1)$ and if pr is a constant $\left(\rho^{2}\right)$, then

$$
\lambda_{n}-\lambda_{n-1}^{A}=2 \rho d_{1}\left(\sigma a_{1}\right)^{-1}+0\left(n^{-2}\right)
$$

where $\sigma$ comes from Corollary 3.4.

Proof. We detail the changes needed to the proof of Theorem 3.5. The end condition (2.6) now gives $\tan \theta(\lambda, 1)=0\left(\lambda^{-1}\right)$, so $\tan \phi(\lambda, 1)=\rho \lambda^{1 / 2} \tan \theta(\lambda, 1)=0\left(\lambda^{-1 / 2}\right)$ where $\rho^{2}=$ (pr)(1). Thus for $\lambda \in I_{n}=\left[\lambda_{n-1}^{A}, \lambda_{n}\right]$,

$$
\begin{aligned}
(\tan \theta(\lambda, 1))_{\lambda} & =-\left(2 \rho \lambda^{3 / 2}\right)^{-1} \tan \phi(\lambda, 1)+\left(\rho \lambda^{1 / 2}\right)^{-1} \phi_{\lambda}(\lambda, 1) \sec ^{2} \phi(\lambda, 1) \\
& \geqq \delta(\rho \lambda)^{-1}+O\left(\lambda^{-3 / 2}\right),
\end{aligned}
$$

by virtue of (3.8). We now estimate $\lambda_{n}$ by using $\tan \theta(\lambda, 1)$ instead of $\cot \theta(\lambda, 1)$, noting that $\tan \theta\left(\lambda_{n-1}^{A}, 1\right)=0$. By the mean value theorem,

$$
\tan \theta\left(\lambda_{n}, 1\right)-\tan \theta\left(\lambda_{n-1}^{A}, 1\right)=\left(\lambda_{n}-\lambda_{n-1}^{A}\right)(\tan \theta(\lambda, 1))_{\lambda}
$$

for some $\lambda \in\left[\lambda_{n-1}^{A}, \lambda_{n}\right]$. Since the left side is $0\left(\lambda_{n}^{-1}\right)=0\left(n^{-2}\right)$ (by the analogue of Corollary 3.4), the first conclusion follows from (5.3).

If $p r=\rho^{2}$ then (3.5) becomes

$$
2 \phi_{\lambda}^{\prime}=\lambda^{-1 / 2}\left[s+o_{1}(1)\right]+\phi_{\lambda} F(x, \lambda)
$$

where $F$ is $0\left(\lambda^{-1 / 2}\right)$ in $L_{1}[0,1]$. Thus the integrating factor $h$ of $(3.6)$ is now $1+0\left(\lambda^{-1 / 2}\right)$ and so

$$
\begin{aligned}
\phi_{\lambda}(\lambda, 1) & =h(0) \phi_{\lambda}(\lambda, 0)+\frac{1}{2} \int_{0}^{1} \lambda^{-1 / 2}\left[s+o_{1}(1)\right] h \\
& =\frac{1}{2} \lambda^{-1 / 2} \sigma+o\left(\lambda^{-1 / 2}\right)
\end{aligned}
$$

Then (5.3) can be estimated more explicitly by

$$
\begin{aligned}
(\tan \theta(\lambda, 1))_{\lambda} & =\left(\rho \lambda^{1 / 2}\right)^{-1}\left(\frac{1}{2} \lambda^{-1 / 2} \sigma\right) \sec ^{2} \phi(\lambda, 1)+0\left(\lambda^{-2}\right) \\
& =\sigma(2 \rho \lambda)^{-1}+0\left(\lambda^{-2}\right)
\end{aligned}
$$

so (2.6) and (5.4) yield the required result, since $\lambda_{n}=0\left(n^{2}\right)$.

Corollary 5.4. If $c_{1}=0<\delta_{1}$ then Corollary 3.6 carries over with the following modifications: $v=0$ if $d_{0}=0, v=-\frac{1}{2}$ if $d_{0} \neq 0$, and $2 \rho d_{1}\left(\sigma a_{1}\right)^{-1}$ must be added to $R$ of (3.10). 
Proof. This follows from Theorem 5.3 and the fact that the RAP now has a righthand Dirichlet condition. The case $p=r \equiv 1$, (hence $\rho=\sigma=1$ ) is discussed by Fulton [4, $\S 4$, Cases 3 and 4$]$.

We conclude with a brief look at the two variable end condition problem. Roughly, this is a combination of the two possibilities corresponding to Theorem 5.1 and Corollary 5.2. The following result is representative and illustrates a rather unusual subcase where (2.1) has no boundary condition at all.

Corollary 5.5. Suppose $\delta_{0}=\delta_{1}=0$, then neither end condition is of Sturm-Liouville type, and that there are no repeated eigenvalues (see the Remark below). Then Theorem 4.2 holds for appropriate $m$ and $M$.

Proof. Arguing as for (5.1), (5.2), we have

$$
\left[a_{j} y(j)-c_{j}\left(p y^{\prime}\right)(j)\right]\left(\lambda+\gamma_{j}\right)=0 \quad j=0,1,
$$

for appropriate $\gamma_{j}$. The first factors provide Sturm-Liouville eigenvalues and the second provide the two "extra" oscillation counts $m$ and $M$ provided $\gamma_{0} \neq \gamma_{1}$. If $\gamma_{0}=\gamma_{1}$, however, both conditions (5.3) are met, so the "extra" oscillation counts come from the two dimensional nullspace of (2.1) with $\lambda=-\gamma_{0}$, but without any boundary conditions.

Remark. Repeated eigenvalues for $j=1$ in (5.3) are as for Theorem 5.2, and those for $j=0$ refer to the left hand asymptotic/Dirichlet problem, unless $\gamma_{0}=\gamma_{1}$ in which case repeated eigenvalues refer to the double asymptotic/Dirichlet problem.

\section{Appendix}

We establish three asymptotic formulae for the eigenvalues $\lambda_{n}$ of the (weighted Sturm-Liouville) problem (2.1) subject to boundary conditions of the form

$$
b_{j} y(j)=d_{j}\left(p y^{\prime}\right)(j),\left(b_{j}, d_{j}\right) \neq(0,0), \quad j=0,1 .
$$

We assume as before that $1 / p, q, r \in L_{1}[0,1]$ with $p, r>0$ and, by scaling (2.1) if necessary, we assume (without loss of generality) that $\int_{0}^{1} 1 / p=1$. Our treatment is related to that of Eastham $[3, \S 4.2]$ for the periodic problem, but our use of (3.2) seems to produce stronger results.

Using the variable

$$
t(x)=\int_{0}^{x} 1 / p
$$

and denoting $d / d t$ by , we see that (2.1) becomes

$$
-\ddot{y}+p q y=\lambda p r y,
$$


where $p, q, r$ and $y$ are viewed as functions of $t \in[0,1]$. The boundary conditions (A.1) then take the form

$$
b_{j} y(j)=d_{j} \dot{y}(j), \quad j=0,1 .
$$

The transformation (3.2) becomes

$$
\cot \phi=(p r \lambda)^{-1 / 2} \dot{y} / y
$$

so we may take (A.1) in the form

where

$$
\cot \phi(\lambda, j)=((p r)(j) \lambda)^{-1 / 2} b_{j} / d_{j}
$$

$$
\phi(\lambda, 0) \in[0, \pi[\text { and } \phi(\lambda, 1)-k \pi \in] 0, \pi]
$$

for some integer $k \geqq 0$. Assume now that $p r \in A C[0,1]$ (as a function of $x$ ). It is easily seen that $p r \in A C[0,1]$ (as a function of $t$ ) and (3.3) then becomes

$$
\dot{\phi}=(\lambda p r)^{1 / 2}-\frac{p q(1-\cos 2 \phi)}{2(\lambda p r)^{1 / 2}}-\frac{(p r) \cdot \sin 2 \phi}{4 p r}
$$

We need two lemmas.

Lemma A.1. Suppose pr, $f \in A C[0,1]$. Then for some constant $C$ depending on $f$,

$$
\left|\int_{0}^{1} f \cos 2 \phi\right| \leqq C \lambda^{-1 / 2} \text { and }\left|\int_{0}^{1} f \sin 2 \phi\right| \leqq C \lambda^{-1 / 2} \text { for } \lambda>1 \text {. }
$$

Proof.

$$
\begin{aligned}
2 \int_{0}^{1} f \cos 2 \phi & =2 \int_{0}^{1} \frac{f \cos 2 \phi}{(\lambda p r)^{1 / 2}}\left(\dot{\phi}+0_{1}(1)\right) \text { by (A.4) } \\
& =\left[\frac{f \sin 2 \phi}{(\lambda p r)^{1 / 2}}\right]_{0}^{1}-\int_{0}^{1}\left(\frac{f}{(\lambda p r)^{1 / 2}}\right) \sin 2 \varphi+0\left(\lambda^{-1 / 2}\right) \\
& =0\left(\lambda^{-1 / 2}\right)
\end{aligned}
$$

The argument for $\int_{0}^{1} f \sin 2 \phi$ is analogous.

Lemma A.2. (i) If $d_{j}=0$ then $\phi\left(\lambda_{n}, j\right)=j(n+1) \pi$.

(ii) If $d_{j} \neq 0$ then $\phi(\lambda, j)=\left(j n+\frac{1}{2}\right) \pi-((p r)(j) \lambda)^{-1 / 2} b_{j} d_{j}^{-1}+o\left(\lambda^{-1 / 2}\right)$, as $\lambda \rightarrow \infty$.

Proof. (i) Since the eigenfunction for $\lambda_{n}$ has $n$ zeros in $] 0,1[$ (cf. Corollary 2.3) the result follows from (A.3). 
(ii) By (A.2), (A.3), $\phi(\lambda, j)=j \mathrm{n} \pi+\cot ^{-1}((p r)(j) \lambda)^{-1 / 2} b_{j} / d_{j}$

$$
=j n \pi+\frac{\pi}{2}+((p r)(j) \lambda)^{-1 / 2} b d_{j}^{-1}\left(\cot ^{-1}\right)^{\prime}\left(\frac{\pi}{2}\right)+o\left(\lambda^{-1 / 2}\right) .
$$

We are now ready for the asymptotic estimates.

Theorem A.3. (i) If $p r \in A C[0,1]$ then $\lambda_{n}^{1 / 2}=\sigma^{-1}(n+v) \pi+o$ (1) as $n \rightarrow \infty$, where $v$ is half the number of Dirichlet conditions specified by $(A .1)$, and

$$
\sigma=\int_{0}^{1}(r / p)^{1 / 2} d x=\int_{0}^{1}(p r)^{1 / 2} d t
$$

(ii) If, in addition, $(p r)^{\prime} \in A C[0,1]$ then $o(1)$ may be replaced by $0\left(n^{-1}\right)$.

(iii) If $\mathrm{pr}$ is a constant $\left(\rho^{2}\right)$ then $0\left(n^{-1}\right)$ may be estimated by

where

$$
((n+v) \pi \rho)^{-1}\left(-[E(j)]_{j=0}^{j=1}+\int_{0}^{1} q / 2\right)+o\left(n^{-1}\right)
$$

$$
E(j)=b_{j} / d_{j} \text { if } d_{j} \neq 0, \quad E(j)=0 \text { if } d_{j}=0 .
$$

Proof. In each case we integrate (A.4) from 0 to $1(d t)$.

(i) From Lemma A.2, the left side is $(n+v) \pi+A$ where $|A|=o(1)$ as $\lambda \rightarrow \infty$. Given $\varepsilon>0$, choose $f \in A C[0,1]$ so that $\left\|f-(4 p r)^{-1}(p r)^{\prime}\right\|_{1}<\varepsilon$. By Lemma A.2, the right side of (A.4) (integrated) becomes

$$
\lambda^{1 / 2} \sigma+B+D
$$

where $|D|<\varepsilon,|B|<\gamma \lambda^{-1 / 2}$, and $\gamma$ depends on $f$ (and hence on $\varepsilon$ ). We now choose $\lambda_{\varepsilon}$ large enough to ensure $|A|<\varepsilon$ and $|B|<\varepsilon$ whenever $\lambda>\lambda_{\varepsilon}$.

(ii) Choosing $f=(4 p r)^{-1}(p r)^{\circ}$, we now obtain from Lemmas A.1 and A.2

$$
(n+v) \pi+0\left(\lambda^{-1 / 2}\right)=\lambda^{1 / 2} \sigma+0\left(\lambda^{-1 / 2}\right) .
$$

Setting

$$
\lambda_{n}^{1 / 2}=\sigma^{-1}(n+v) \pi+\eta
$$

we have $\eta=o(1)$ from (i), so $0\left(\lambda^{-1 / 2}\right)=0\left(n^{-1}\right)$ and the conclusion follows from (A.5).

(iii) Refining (A.5), we have from Lemmas A.1 and A.2

$$
(n+v) \pi-\left[\lambda^{-1 / 2} \rho^{-1} E(j)\right]_{j=0}^{j=1}=\lambda^{1 / 2} \sigma-\int_{0}^{1}\left(2 \rho \lambda^{1 / 2}\right)^{-1} p q d t+o\left(\lambda^{-1 / 2}\right)
$$


so using (A.6) again, we obtain

$$
((n+v) \pi \rho)^{-1} \sigma\left(-[E(j)]_{j=0}^{j=1}+\int_{0}^{1} 2^{-1} q d x\right)=\eta \sigma+o\left(n^{-1}\right)
$$

Remark. It is possible to use bounded variation instead of absolute continuity at various points, cf. $[1, \S 12.8],[5, \S 8.4]$.

\section{REFERENCES}

1. F. V. Atkinson, Discrete and Continuous Boundary Problems (Academic Press, 1964).

2. F. V. Atkinson and A. B. Mingarelu, Asymptotics of the number of zeros and of the eigenvalues of general weighted Sturm-Liouville problems, J. Reine Angew. Math. 375/6 (1987), 380-393.

3. M. S. P. Eastham, The Spectral Theory of Periodic Differential Equations (Scottish Academic Press, 1973).

4. C. T. Fulton, Two-point boundary value problems with eigenvalue parameter contained in the boundary conditions, Proc. Roy. Soc. Edinburgh 77A (1977), 293-308.

5. E. Hille, Lectures on Ordinary Differential Equations (Addison Wesley 1969).

6. D. B. Hinton, An expansion theorem for an eigenvalue problem with eigenvalue in the boundary condition, Quart. J. Math. Oxford, 30 (1979), 33-42.

7. E. L. INCE, Ordinary Differential Equations (Dover, 1956).

8. H. LANGer and A. SChNeider, On spectral properties of regular quasidefinite pencils $F-\lambda G$, preprint.

9. J. Poschel and E. TRubowitz, Inverse Spectral Theory (Academic Press, 1987).

10. W. T. REID, Ordinary Differential Equations (J. Wiley, 1971).

11. W. T. Reid, Sturmian Theory for Ordinary Differential Equations (Springer-Verlag, 1980).

12. J. Walter, Regular eigenvalue problems with eigenvalue parameter in the boundary conditions, Math. Z., 133 (1973), 301-312.

Department of Mathematics AND STatistics UNIVERSITY OF CALGARY Calgary Alberta T2N 1N4 CANADA
Department of Mathematics UNIVERSITY OF SASKATCHEWAN SASKatOON SASKATCHEWAN S7N OW0 Canada
Department of Mathematics UNIVERSITY OF SHIRAZ IRAN 71454 